\title{
Effect on magnetic sensors and shape identification of 3d non-axisymmetric eddy currents in ITER
}

\section{R. Albanese, G. Ambrosino, A. Pironti ${ }^{1}$, G. Rubinacci}

DIETI, Università Federico II di Napoli

Via Claudio 21, 80125, Napoli, Italy

E-mail: \{raffaele.albanese, ambrosin, pironti, rubinacci\}aunina.it

M. Mattei

DIII, Seconda Università di Napoli

Via Roma 29, Aversa (CE), Italy

E-mail: massimiliano.matteicunina2.it

\section{S. Ventre, F. Villone}

DIEI; Università di Cassino e del Lazio Meridionale

Via Di Biasio 43, 03043, Cassino (FR), Italy

E-mail: \{ventre, villone\}aunicas.it

\section{S. Arshad}

Fusion for Energy

Torres Diagonal Litoral B3 - c/ Josep Plá n.2 Barcelona, Spain

E-mail: Shakeib. Arshadef4e.europa.eu

\section{G. Vayakis}

ITER Organization

R.te de Vinon sur Verdon, CS 90 046, 13067 St Paul Lez Durance Cedex, France

E-mail: George.Vayakiseiter.org

\begin{abstract}
A simplified procedure is proposed for the evaluation of the effects on magnetic measurements and on plasma shape identification of non-axisymmetric eddy currents induced in conducting structures by fast plasma events. The procedure has been successfully applied to several events in ITER.
\end{abstract}

First EPS Conference on Plasma Diagnostics - $1^{\text {st }}$ ECPD

14-17 April 2015,

Villa Mondragone, Frascati (Rome) Italy

${ }^{1}$ Speaker 


\section{Introduction}

Tokamaks are fusion devices which are ideally axisymmetric. However, due to the necessity of accessing the interior of the torus, several deviations from axial symmetry are unavoidable in the conductors surrounding the plasma (ports, segmentation of TF coils etc.). As long as these effects can be considered as relatively small perturbations, the design of future devices like ITER can be carried out supported by $2 \mathrm{D}$ (axisymmetric) models and numerical codes.

This is for instance the case of magnetic feedback control, for which usually 2D codes are used [1]. In particular, for what concerns the plasma shape control, the definition of the plasma boundary is usually made by identification codes, which use the available magnetic measurements. In particular, the EFIT code [2] solves the GradShafranov equations, choosing the degrees of freedom of the MHD equilibrium so as to best fit the magnetic measurements at given positions (where the pick-up coils and saddle loops are located) and other non-magnetic diagnostics (e.g. MSE).

In order to take into account three-dimensional effects using the same approach, in principle one should resort to complicated 3D plasma equilibrium models able to account for 3D conductors. This paper proposes instead a simplified approximate approach, which computes the effect on magnetic measurements of eddy currents induced in 3D structures by fast transients (e.g. H-to-L transition, VDEs, etc.) of an axisymmetric plasma, described in terms of equivalent toroidal filaments. For this purpose, the CARIDDI [3] eddy current code is used. Such 3D perturbations on magnetic measurements are then fed into EFIT, in order to evaluate the effect on the shape identification.

The paper is organized as follows. Section 2 describes the procedure proposed to evaluate the plasma shape deformations due to $3 \mathrm{D}$ eddy currents flowing in the non axial-symmetric structures, and the gap estimation errors obtained using the EFIT++ equilibrium reconstruction code. Section 3 describes the application of the proposed procedure to several cases. Finally, Section 4 presents the conclusions of the analyses in terms of plasma-wall gap estimation error deterioration.

\section{The proposed simplified procedure}

The procedure used for the analysis is based on the assumption that the 3D asymmetries of the structure give rise to small deformations of the plasma shape in the various poloidal sections, so that the plasma contribution to the flux map remains practically unchanged.

The procedure consists of the following steps.

1. STEP 1. For a specified plasma disturbance (e.g. VDE, minor disruption, ...) or scenario segment (e.g. L-H transition, plasma current ramp, ...) determine the plasma time evolution using the CREATE_NL axial-symmetric simulation code [4].

2. STEP 2. Approximate the plasma movements and the plasma current distribution time evolution by means of a dense distribution of time-varying filamentary currents located so as to fill up the whole poloidal plasma region.

3. STEP 3. Calculate the eddy currents time evolution in the non axial symmetric structures by using the CARIDDI code [3], and using as inputs the currents in the active coils and in the filaments which approximate the plasma. 
4. STEP 4. For a number of poloidal sections in the considered sector, at each time instant of the considered time evolution, determine the flux and field maps as superposition of the following contributions:

- $\quad$ the active coil currents

- $\quad$ the axisymmetric plasma filaments

- $\quad$ the non axial symmetric eddy currents

5. STEP 5. For each of the above considered poloidal sections, using the flux and field maps, calculate the time evolution of the simulated magnetic measurements and of the plasma shape (defined as the last closed flux line).

6. STEP 6. For each of the above considered poloidal sections, using the EFIT++ code [2], determine the time evolution of the gap estimation errors, without using any information about the eddy currents.

It should be remarked that the calculation of the plasma shape is approximate: in the presence of 3D plasma deformations, the plasma shape can no longer be calculated as the last closed flux line (which is, in any case, axisymmetric), but it should be calculated as the last closed magnetic surface.

We also stress that the contribution of the plasma to the magnetic sensor measurements is kept unchanged after the shape deformations. Hence the simulated sensor measurements used in next sections for gap estimation take into account the contribution due to 3D eddy currents, but they do not take into account the correct information on the plasma deformation.

\section{Results}

The procedure proposed in the previous section has been applied to ITER. In particular, three plasma events have ben considered: L-H transition, plasma current ramp-down, Vertical Displacement Event (VDE). Indeed, such events are expected to induce significant eddy currents in the structures and hence can potentially highlight significant 3D deviations. Fig. 1 shows the reference plasma shape and the gaps used by CREATE_NL to define the plasma shape. Fig. 2 shows instead the 3D meshes used by the CARIDDI code in the computation of the eddy currents: the first one mimics an axisymmetric situation and is used mainly for benchmarking; the second one instead includes several details about the conducting structures (ports, blankets, divertor etc.). The meshes span $40^{\circ}$ in the toroidal direction; rotational symmetry is adopted to recover the full torus.

The plasma has been simulated in CARIDDI with around 10000 toroidally axisymmetric filaments, located in the plasma region depicted in Fig. 1, with a time varying current, so as to reproduce the actual behaviour predicted by CREATE_NL evolution. The outputs provided by CARIDDI consist of the simulated magnetic measurements (magnetic fields and fluxes) at the positions where ITER sensors are located. A sensitivity analysis on the toroidal location of these sensors has been carried out, showing no significant differences. These simulated measurements are then fed into EFIT so as to identify the plasma boundary and estimate the gaps. Comparing these estimated gaps with the nominal ones provided by CREATE_NL, the gap estimation error can be evaluated. 
Figure 3 shows the gaps estimated by EFIT for the L-H transition, with the two 3D meshes reported in Fig. 1; the differences are around some mm. Figure 4 reports two sample simulated magnetic measurements for the plasma current ramp-down case, highlighting that the main 3D effects are expected on the fields and fluxes in the outboard region. Figure 5 shows the gap reconstruction error for the VDE event, which shows that the expected error is of the order of 1 $\mathrm{cm}$ and is similar when using the 3D axisymmetric mesh and when using the 3D not axisymmetric mesh.

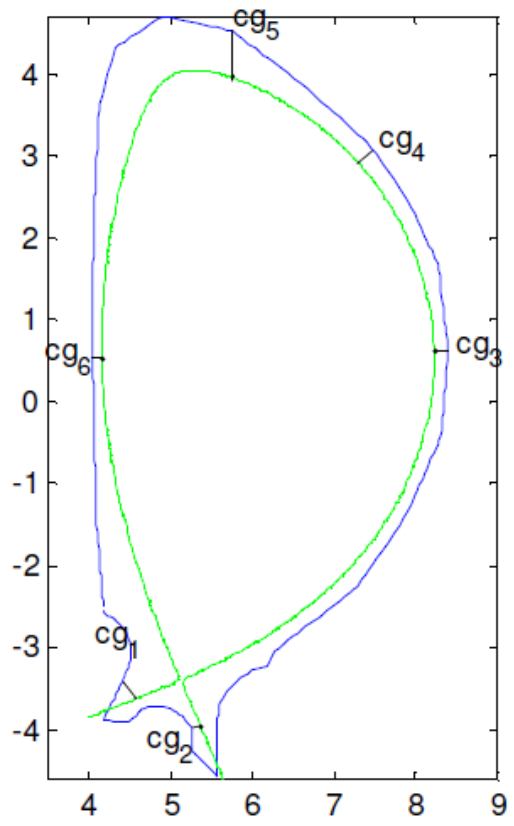

Fig. 1. Plasma shape and gap definition.
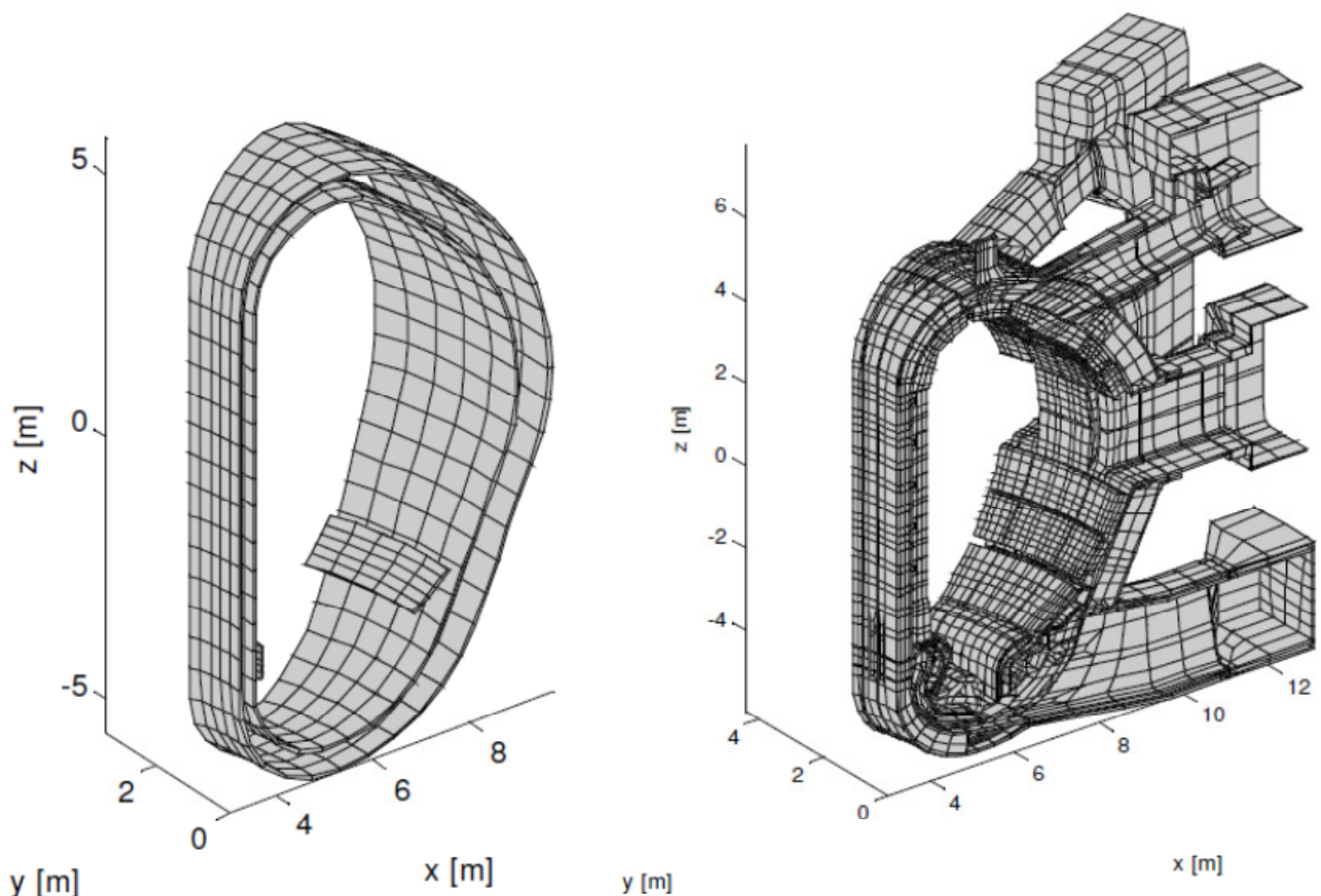

Fig. 2. 3D meshes used in the analysis. 

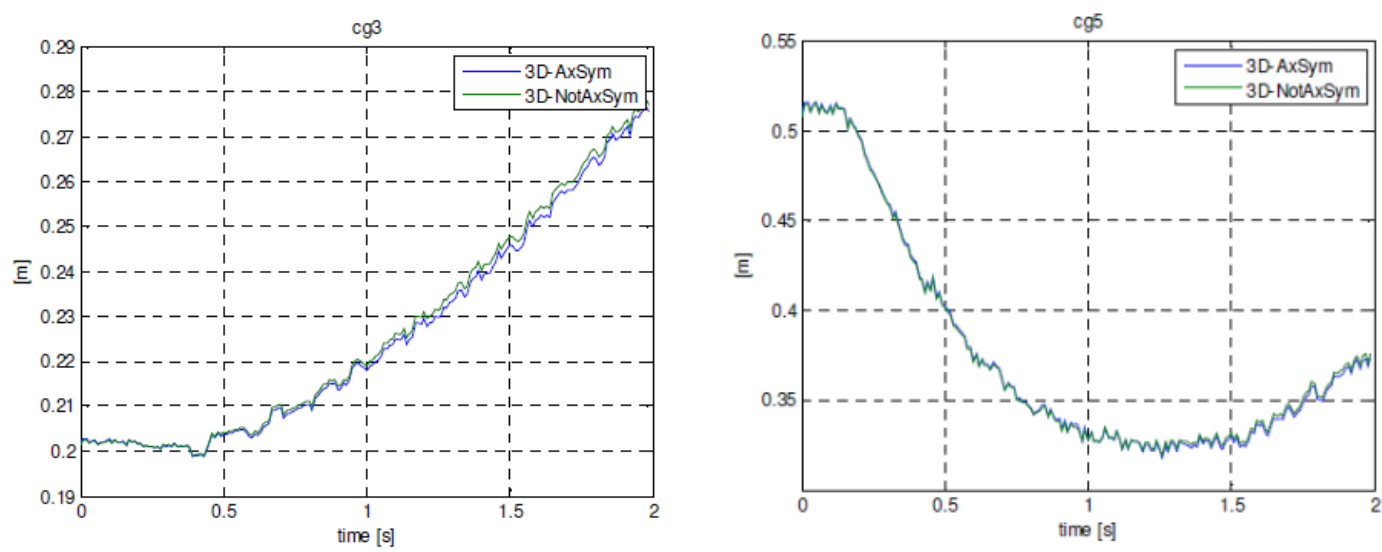

Fig. 3. Gap estimated by EFIT using two different meshes (L-H transition)
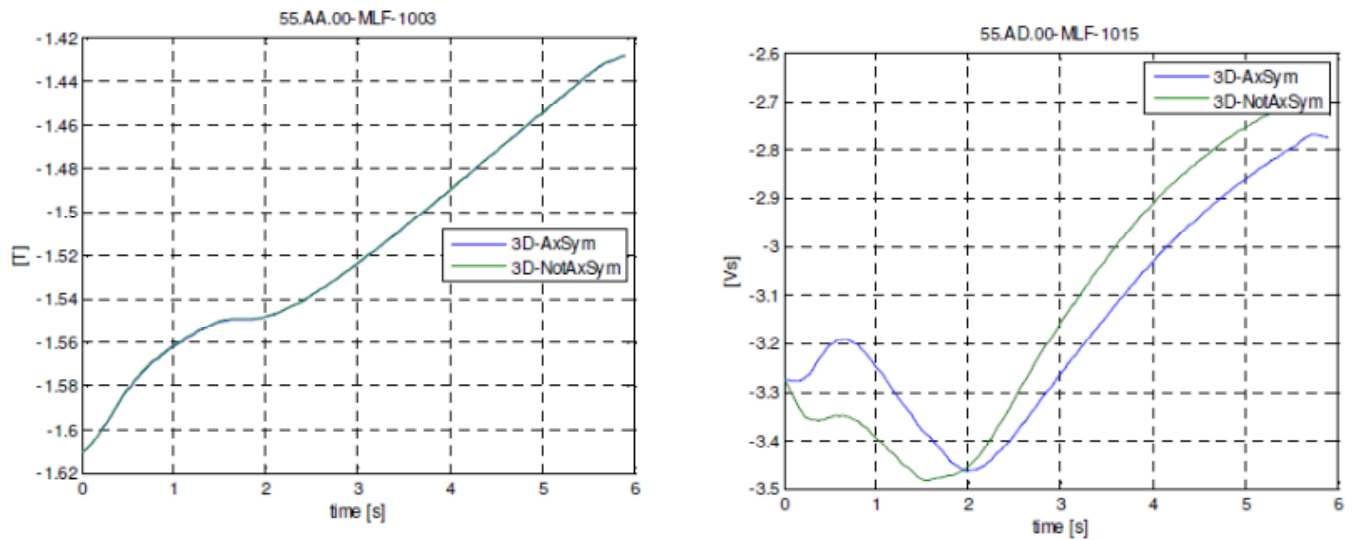

Fig. 4. Simulated measurements (plasma current ramp-down): (a) tangential magnetic field pickup in the inboard side, (b) flux saddle loop on the outboard side
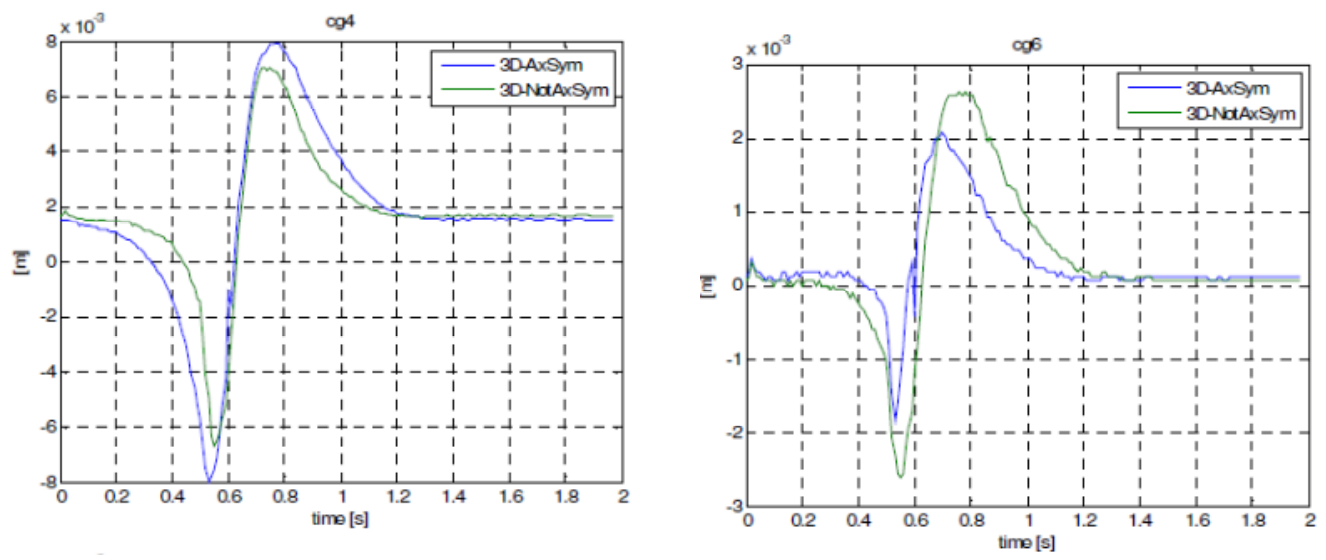

Fig. 5. Gap estimation errors (VDE). 


\section{Conclusions}

The proposed simplified procedure allowed us to estimate the expected effect on magnetic sensors and shape identification in ITER of $3 \mathrm{~d}$ non-axisymmetric eddy currents induced by specific plasma events ( $\mathrm{L}-\mathrm{H}$ transition, plasma current ramp-down, VDE), which are among the most critical disturbances in terms of eddy currents induced in the passive structures. The main conclusions are that the non axial symmetry of the eddy currents flowing in the non-axial symmetric structures does not significantly modify the plasma shape (a few $\mathrm{mm}$ on the outboard gaps) and does not significantly modify the sensor measurements, except for the outboard pickup coils (about $3 \mathrm{mT}$ ) and saddle coils (about $0.15 \mathrm{Vs}$ ).

The work reported in this paper was funded partially Italian MIUR under PRIN grant 2010SPS9B3 and partially by Fusion for Energy under Grant F4E-GRT-047. This publication reflects the views of the authors only. Fusion for Energy cannot be held responsible for any use which may be made of the information contained herein.

\section{References}

[1] R. Albanese and F. Villone, Nucl. Fusion 38 (1998) 723

[2] J.R. Ferron et al. Nucl. Fusion 38 (1998) 1055

[3] R. Albanese, G. Rubinacci, Adv. Imaging El. Phys. 102 (1998) 1-86

[4] R. Albanese, G. Calabrò, M. Mattei, F. Villone, Fus. Eng. Des. 66-68 (2003) 715-718 